\title{
The Patient History Project: Medical Student Innovation in Improving Medical Care for Asylum Applicants
}

\author{
Jennifer N. Angell", BS, MD; Jack H. Buchanan ${ }^{\dagger}, \mathrm{MD}, \mathrm{PhD}$; \\ Thomas Rogers ${ }^{\ddagger}, \mathrm{BA}$; Mary Ellen Michele Heisler ${ }^{\S}, \mathrm{MD}, \mathrm{MPH}$
}

\section{Background}

Persons who experienced persecution in their home country may apply for asylum with the United States. This population has complex medical needs complicated by cultural and linguistic barriers. This report outlines the design and evaluation of the Patient History Project (PHxP), a collaborative program between the University of Michigan Asylum Collaborative (UMAC) and a local non-profit, Freedom House Detroit (FHD), to improve the quality of health care for asylum-seekers while training medical students to work with vulnerable populations.

\section{Methods}

In the PHxP, medical students trained by UMAC interviewed FHD residents and created electronic medical records that residents could share with subsequent medical providers. These sessions additionally provided an opportunity for asylum-seekers to rehearse health care utilization in the comfort of their home. Surveys of students and asylum-seekers, and a semi-structured interview with FHD staff, were conducted to evaluate the program's performance on multiple dimensions.

\section{Results}

Asylum-seekers reported high levels of overall satisfaction with $\mathrm{PHxP}$ and increased confidence in the health care setting. Medical student volunteers reported gaining cultural sensitivity and clinical skills. FHD staff noted greater insight into resident medical needs.

"University of Michigan Medical School, angell.jenn@gmail.com

tUniversity of Michigan Medical School

${ }^{\ddagger}$ Freedom House Detroit

§University of Michigan, mheisler@umich.edu

doi: 10.3998/mjm.1433

Conflicts of interest:

The authors have no conflicts of interest to disclose 


\title{
Conclusions
}

As the number of asylum-seekers in the United States continues to rise, so will the need of specialized services including medical care. This program is one strategy to help address the medical needs of asylum-seekers. Medical schools with programs to provide forensic medical exams and affidavits for asylum-seekers would be particularly well situated for the trial of such a program.

\author{
Keywords \\ asylum $\cdot$ refugee $\cdot$ Detroit
}

\section{Introduction}

The high prevalence of international conflict, natural disasters, and consequent diasporas have led to an increase in the need in recent years for medical professionals trained to care for displaced persons. ${ }^{1,2}$ Community service learning has been identified as an effective means of teaching health professional students the realities of health disparities and how to address them at an individual level., The University of Michigan Asylum Collaborative (UMAC) is a medical student organization that utilizes service learning to benefit the population of persecuted persons seeking asylum in the state of Michigan. Individuals can qualify for asylum in the United States if they have suffered persecution in their home countries on account of their race, religion, nationality, social group, or political opinion. ${ }^{5}$ The majority of UMAC's clients are residents of Freedom House Detroit (FHD), a multidimensional organization that provides housing as well as social and legal services to asylum-seekers. ${ }^{6}$ UMAC's medical student and physician volunteers undergo training to conduct forensic medical evaluations. Evidence of bodily and/or psychological harm is recorded in a medical affidavit submitted with the federal application for asylum. Interviews with asylum lawyers by our group have reinforced the critical importance of the medical affidavit for establishing claims for asylum, and nationwide estimates suggest that credible asylum applications that include such an affidavit are more than twice as likely to succeed. ${ }^{7,8}$

At our institution, there is overwhelming medical student interest in assisting asylumseekers. Although caseloads increase each year, to date UMAC is unable to accommodate all interest through participation in forensic medical examinations. We identified the improvement of medical care as an additional need of this community, which could be supplemented by medical students while providing a valuable learning opportunity. Asylum-seekers have often experienced physical and psychological trauma that frequently results in long-term health sequelae that require sensitive care. ${ }^{9}$ An additional challenge asylum-seekers face is the cultural and linguistic barriers to effectively communicating with health care providers. ${ }^{10,11}$ Most asylum-seekers lack health insurance, so those residing at FHD receive care at various free clinics. Due to institutional limitations, UMAC is not able to host a medical clinic specifically for asylum-seekers.

We created the Patient History Project (PHxP) to address the needs of our local community of asylum-seekers while engaging medical students in unique service learning. The PHxP aims to improve FHD residents' ability to communicate with providers during subsequent health care visits by creating a familiar "rehearsal" environment for their first encounter and an initial 
record to facilitate future care interactions. From the viewpoint of FHD staff, such a program had the potential to increase clients' confidence in navigating the US health care system, as residents often have particular difficulty communicating sensitive needs (eg, gynecological visits for a rape survivor), trusting authority given their vulnerable legal status, and overcoming linguistic and cultural barriers. The PHxP also had the potential to facilitate a broader organizational effort by the FHD to consolidate and streamline resident medical information. Since the majority of residents' health care is provided by at least two local non-profit clinics with which FHD partners, having a centralized in-house system of electronic medical record-keeping could afford FHD more efficiency and autonomy in tracking and managing resident needs.

To meet these needs, the PHxP recruits medical student volunteers to interview FHD residents and document their medical histories in a free, secure online electronic medical record (EMR) that can be shared with health care providers during subsequent medical appointments. FHD staff are also provided access to the EMR for review, discretionary sharing with health care providers and/or FHD residents directly, and to update after residents' medical appointments. As an educational tool, the $\mathrm{PHxP}$ aims to provide preclinical medical students the opportunity to practice clinical history taking, documentation skills, and, when necessary, medical interpretation while gaining experience working with diverse and vulnerable populations.

A secondary aim of the program is to evaluate and improve upon its strengths and weaknesses so that it may be replicated in other programs for asylum-seekers and other recent immigrants and refugees. The current article has three objectives: (1) to describe the PHxP's development, implementation, and evaluation efforts to date; (2) to summarize the experiences of participants and stakeholders (ie, students, residents, and FHD staff) as evaluated by surveys and semi-structured interviews; and (3) to use the evaluations data to understand and reflect upon key program strengths and areas for improvement.

Based on our initial design goals and observations from multiple iterations of the program, we predicted the PHxP's strongest performance areas to be enhancing student volunteers' cultural sensitivity, increasing resident-participant confidence in future medical encounters, and improving staff responsiveness to resident medical needs. Conversely, we predicted its weakest performance areas to be student volunteer's perceptions of benefit for resident-participants, resident-participants' avoidance of reliving past trauma, and reducing administrative burden on staff.

\section{Methods}

\section{Program Design}

A project proposal was created in collaboration between Freedom House Detroit's (FHD) community outreach coordinator and the FHD program manager. The proposal was reviewed and approved by the UMAC board of directors and the FHD director. Student volunteers signed a confidentiality agreement, and FHD resident-participants signed a release of information to allow their EMR to be shared with select FHD staff and appropriate free clinic providers upon scheduling of appointments.

The program consists of monthly sessions held at FHD during which University of Michigan medical students interview residents to elicit and document their past medical histories in an EMR. In the monthly session, two teams of preclinical students interview FHD residents in preparation for future health care visits. Each team of two students interviews a resident to collect a thorough past medical history. During the interview, one student leads the history taking while the other documents the history in the EMR. Students switch roles for the second round 


\begin{abstract}
is a 23 year old woman with a past medical history of cataract surgery and sinusitis. She reports feeling sad, hopeless, with poor interest in activities she used to have pleasure in beforehand, difficulty sleeping with nightmares, poor appetite, and difficulty concentrating. Depression screening tool was assessed and patient is PHQ-2+, indicative of clinical depression. She feels safe in her current home.

\section{Recommendations:}

1. Depression: should follow-up with a primary care physician and/or mental health specialist to address her ongoing symptoms of depression. She is positive on our screening tool for depression, and this needs further medical review and may require medical intervention, such as medication. She feels safe in her current home.

2. Sinusitis: previously used a sinus nasal spray that worked for her. These can be acquired over the counter at any pharmacy with some brands, such as Flonase.

3. Cataracts: Previous medical problem resolved with surgery in 2012. She should have annual eye exams.

4. Vaccination: is unclear on her current vaccination status and should be seen by a physician for blood titers and vaccine boosters if necessary.

5. Headaches, fatigue, dizziness: these are symptoms that can be associated with anemia. should be seen by a physician for basic blood work and treatment of anemia if needed.

6. Women's health: has never received any preventative gynecologic care. She should see a physician to establish care and receive routine pap smears. She also expresses concern regarding her infrequent periods and wishes this to be addressed.

7. Trouble sleeping and leg spasms: This might be associated to restless legs, a problem that can be very bothersome and should be assessed by a physician.
\end{abstract}

Figure 1. Example Medical Summary.

Summary of asylum-seeker's medical history compiled by the supervising clinical medical student, approved by the faculty physician advisor, and shared with Freedom House Detroit.

of interviews. Non-English-speaking participants (most often francophone) are paired with at least one appropriate bilingual student interviewer who interprets into English for the other student to transcribe in the EMR.

Immediately after an interview, the preclinical team presents to the supervising clinical student. This practice serves several functions: it provides practice with patient presentations for the preclinical student, serves as a safety mechanism to determine if there are any remaining clinical questions, and provides an opportunity to discuss any urgent medical concerns or sensitive topics such as self-harm or sexual health. The clinical student advises the preclinical students, who then return to the interview to address any remaining concerns.

After the completion of the session, the clinical student is responsible for documenting a summary to be provided to FHD staff. The summary document comprises an individual summary for each resident detailing relevant past medical history, primary medical concerns at the time, and a section highlighting recommended follow-up for identified needs, including health maintenance and vaccinations (Figure 1). The summary is shared with select FHD staff who review it and schedule follow-up appointments as necessary. The EMR can be printed for the resident to bring to his or her medical appointment.

\title{
Program Evaluation
}

A 16-question electronic survey was distributed to 24 student volunteers, and a 10-question paper survey was distributed to 5 FHD resident-participants. Both the student volunteers and resident-participants were asked to rate various aspects of their experiences with the $\mathrm{PHxP}$ 
with a Likert scale ranging from 1 (completely disagree) to 5 (completely agree), closed questions, and free response questions. A 1-hour, semi-structured evaluation interview was conducted with the FHD case manager to reflect on the program and identify key administrative challenges.

\section{Results}

Over a 9-month period in 2016, the PHxP created an EMR for 26 individual FHD residents across 7 separate visits. Each visit included 2 to 5 preclinical medical students and 1 to 2 clinical medical students. Over this period, 30 different medical students participated in history taking (21 preclinical, 9 clinical); 7 of these students also served as French interpreters.

\section{Resident-Participants}

Only 5 of the 26 resident-participants were still living at Freedom House and available to take the survey at the time of its distribution. The resident-participants reported high levels of overall satisfaction with the $\mathrm{PHxP}(4.60 \pm 0.89$ on a 5 -point scale). They reported increased confidence in the health care setting $(4.60 \pm 0.89)$ and increased efficiency in subsequent health care interactions $(4.50 \pm 1.00)$. Results on all 10 questions from the resident-participant survey are summarized in Table 1.

Table 1. Freedom House Detroit Resident Responses Rated on a Scale of 1 (completely disagree) to 5 (completely agree) $(\mathrm{N}=5)$

\begin{tabular}{|l|c|c|c|}
\hline & n & Mean & SD \\
\hline Summary & & & \\
\hline Overall satisfaction with the PHxP & 5 & 4.60 & 0.89 \\
\hline I understood the purpose of the PHxP and my participation in it & 4 & 4.25 & 0.96 \\
\hline Reasons for participation & & & \\
\hline Gain more confidence in health care settings & 5 & 4.80 & 0.48 \\
\hline Make future health care interactions more efficient & 5 & 4.80 & 0.48 \\
\hline Avoid having to retell story or describe health concerns & 5 & 4.60 & 0.55 \\
\hline Receive more comprehensive or focused health care & 5 & 4.40 & 0.55 \\
\hline For my own records & 5 & 3.80 & 1.64 \\
\hline FHD staff recommended I participate & 5 & 2.80 & 2.05 \\
\hline Reported outcomes & & & \\
\hline Avoided having to retell story or describe health concerns & 5 & 4.80 & 0.45 \\
\hline Gained more confidence in health care settings & 5 & 4.60 & 0.89 \\
\hline Made subsequent healthcare interactions more efficient & 4 & 4.50 & 1.00 \\
\hline Received more comprehensive or focused health care & 5 & 4.00 & 1.41 \\
\hline Participation has influenced my relationship with US health care & 5 & 4.20 & 1.30 \\
\hline
\end{tabular}




\section{Student Volunteers}

Of the 24 medical students who volunteered, 12 completed a questionnaire (50\% response rate) regarding their experiences (Table 2). Six of the 12 students had previously participated in at least one forensic medical evaluation of an asylum-seeker resulting in a medical affidavit. For 5 of the 12 students, the PHxP was their first experience working with asylum-seekers. Notably, students reported gaining insight into the experience of asylum-seekers $(4.50 \pm 0.52)$, learning the importance of cultural sensitivity as a health care provider $(4.42 \pm 0.51)$, and gaining clinical skills (4.17 \pm 0.58 ). Student respondents' perceptions regarding the effectiveness of the $\mathrm{PHxP}$ in fulfilling needs of the residents $(3.92 \pm 0.67)$, and of the positive impact of their involvement $(3.67 \pm 1.07)$, were less positive and more variable.

Students' free responses were thematically coded and counted. The most cited learning outcomes were increased awareness of sociocultural and other issues specific to asylum care $(n=5)$; improved history taking and documentation skills $(\mathrm{n}=2)$; improved leadership and management skills $(\mathrm{n}=2)$; making meaningful human connections $(\mathrm{n}=2)$; and increased interest in asylum work $(\mathrm{n}=2)$. The most cited participant outcomes were establishment of a thorough medical record $(n=4)$; consolidation of information to facilitate future reference $(n=2)$; and the participant's voice being heard $(n=1)$. Finally, in terms of improvements to the program, one student suggested offering a medical French course, and one proposed that more attending-level teaching be incorporated.

\section{Freedom House Detroit Staff}

A semi-structured interview was conducted with the FHD case manager. The case manager identified two key strengths of the project from a staff perspective. First, the opportunity to "rehearse" the medical encounter in the safe and familiar environment of FHD did appear to enhance residents' confidence and proficiency with relevant vocabulary, norms, and expectations. Second, in some instances, resident-participants divulged important information previously unknown to FHD staff. For example, a PHxP history elicited needs in the management of a resident's diabetes mellitus, which had not been elicited during his intake interview.

Table 2. Student Volunteer Responses Rated on a Scale of 1 (completely disagree) to 5 (completely agree) $(\mathrm{N}=12)$

\begin{tabular}{|l|c|c|c|}
\hline & $\mathbf{n}$ & Mean & SD \\
\hline "I would participate again" & 12 & 4.67 & 0.49 \\
\hline "I gained insight into lives of asylum-seekers" & 12 & 4.50 & 0.52 \\
\hline "I understood program goals" & 12 & 4.42 & 0.67 \\
\hline "I learned importance of provider's cultural sensitivity" & 12 & 4.42 & 0.51 \\
\hline "I gained clinical skills" & 12 & 4.17 & 0.58 \\
\hline "My preparation was adequate" & 12 & 3.92 & 0.67 \\
\hline "I think the PHxP was effective" & 12 & 3.92 & 0.67 \\
\hline "I am more likely to work with asylum-seekers again" & 12 & 3.75 & 0.87 \\
\hline "I had a positive impact on residents" & 12 & 3.67 & 1.07 \\
\hline
\end{tabular}


The case manager also identified the most important weakness of the PHxP: its inability to facilitate, as was hoped, a broader organizational effort to consolidate and streamline resident medical information. Personnel and resource constraints limited FHD's ability to liaise directly with their medical clinic partners, or with UMAC, on medical issues, causing the PHxP to complexify the organization's existing challenges in administering resident health care.

\section{Discussion}

The number of asylum-seekers in the United States increased almost 150\% between 2014 and 2017 , reflecting a significant population in need of specialized services including medical care. ${ }^{2}$ The Patient History Project (PHxP) was created to address medical needs in the asylum community at Freedom House Detroit (FHD) through medical student service learning. The PHxP created electronic medical records for 26 FHD residents, providing them with a tool to facilitate communication with subsequent providers as well as a safe space to gain familiarity and confidence with medical encounters. It also provided medical student volunteers with opportunities to elicit, interpret, document, and triage medical concerns within a uniquely sensitive, multicultural patient population.

Overall, our limited results suggest that the $\mathrm{PHxP}$ accomplished its goal of increasing FHD residents' confidence in interacting with the US health care system while engaging medical students in valuable service learning with asylum-seekers. In terms of program strengths from the resident-participant perspective, contrary to our hypothesis, residents rated the avoidance of past traumas through PHxP participation as the strongest positive outcomes $(4.80 \pm 0.45)$. More consistent with our hypothesis, resident-participant surveys highlighted increased confidence with subsequent medical encounters as the most important reason for participating $(4.80 \pm 0.48)$ and second-most positive outcome $(4.60 \pm 0.89)$. In terms of weaknesses, participants expressed weaker and more variable perceptions about improvements in the quality of subsequent care $(4.00 \pm 1.41)$. This was likely because resource, personnel, and institutional constraints on the part of FHD and its partner clinics ultimately limited the extent to which the EMR documents created by the PHxP could be utilized in subsequent encounters.

Results of the student volunteer survey were consistent with our hypothesis in that the highest-rated outcomes were gaining insight into the experience of asylum-seekers $(4.50 \pm$ 0.52 ) and learning the importance of cultural sensitivity as a health care provider $(4.42 \pm 0.51)$, while the lowest-rated (and more variable) outcomes were effectiveness of the PHxP in fulfilling needs of the residents $(3.92 \pm 0.67)$ and the positive impact of their involvement $(3.67 \pm$ 1.07). Free response data corroborated the importance of increased sensitivity to cultural and asylum-related issues $(n=5)$ but also revealed significant support for the PHxP's benefit to resident-participants $(n=7)$, emphasizing the variability among student views about the latter. Overall, these results affirmed a primary objective of the program in providing medical students with service learning opportunities to understand the special cultural, linguistic, and psychological needs of asylum-seekers. They also reveal uncertainty among students about the program's efficacy and overall benefit to participants. This uncertainty likely owes to the limited scope of the student role and to the fact that at the time of their involvement, administrative infrastructure was not yet in place to demonstrate continuity of the initial EMR created, so it was not initially clear what its ultimate impact would be.

Consistent with our hypothesis about the staff perspective, the case manager's primary critique of the PHxP was its inability to overcome or circumvent FHD's underlying personnel and resource constraints, or to liaise directly with their clinic partners, causing the $\mathrm{PHxP}$ to complicate the administration of resident health care. Exacerbating this, the free online EMR 
utilized by the PHxP had privacy and accessibility limitations. To enhance health literacy and personal agency of participants, a suitable EMR should include a patient portal or other feature that provides participants with a means of accessing and interacting with their individual record for personal reference and future updates.

Despite the resident-participants not highlighting it as a main concern, an important inherent limitation of programs such as the $\mathrm{PHxP}$ is their potential to effect vicarious psychological trauma. Even though $\mathrm{PHxP}$ volunteers were explicitly trained to avoid eliciting residents' histories of abuse or torture, it was often impossible to completely avoid such details in the setting of comprehensive medical history taking. To maximize the value of such a program for asylum-seekers, it is thus vital to determine the proper balance between the medical "rehearsal" benefit and the risk of reliving past trauma and to manage that risk with adequate volunteer training and other trauma-informed best practices.

Global limitations to the interpretation of our data include a small available sample size, especially of the resident-participants, and the subjectivity and single time point of the outcome measures. Future evaluation design should conduct participant surveys before and after participation in the program, include a control group, and/or establish more objective outcome measures, eg, rates of health care utilization of asylum-seekers after participating in the $\mathrm{PHxP}$.

\section{Conclusion}

The Patient History Project represents one medical student organization's innovative efforts to enhance the quality of health care for asylum-seekers in the United States. Given the rising number of persons seeking asylum within the United States, such a program could be an important mechanism for helping to address the medical needs of this population. The adoption of similar programs by other medical schools would increase the number of asylum-seekers receiving individually tailored medical support during the stressful process of seeking asylum. Medical schools with programs to provide forensic medical exams and affidavits for asylum-seekers would be particularly well situated for the trial of such a program.

\section{Acknowledgments}

The authors would like to thank Freedom House Detroit for their tireless work on behalf of asylumseekers and for partnering with the University of Michigan Asylum Collaborative on the implementation of the Patient History Project. The authors would additionally like to thank Genevieve Allen and Mariam Salman for their contributions to the development of the Patient History Project. There is no funding or financial support to declare.

\section{References}

1. Stone H, Choi R, Aagaard E, et al. Refugee health elective. MedEdPORTAL Publ. 2013;(9). doi:10.15766/mep_2374-8265.9457

2. Mossaad N. Annual Flow Report: Refugees and Asylees: 20I7. US Department of Homeland Security; 2019.

3. Cené CW, Peek ME, Jacobs E, Horowitz CR. Community-based teaching about health disparities: combining education, scholarship, and community service.J Gen Intern Med. 2010;25(suppl 2):S130-S135. doi:10.1007/s11606-009-1214-3

4. Yoder KM. A framework for service-learning in dental education. J Dent Educ. 2006;70(2): 115-123. http://www.ncbi.nlm.nih.gov/pubmed/16478925 
5. Asylum. US Citizenship and Immigration Services. Accessed July 10, 2018. https://www.uscis. gov/humanitarian/refugees-asylum/asylum

6. Freedom House Detroit. https://freedomhousedetroit.org

7. Scruggs E, Guetterman TC, Meyer AC, VanArtsdalen J, Heisler M. “An absolutely necessary piece": a qualitative study of legal perspectives on medical affidavits in the asylum process. J Forensic Leg Med. 2016;44:72-78. doi:10.1016/j.jflm.2016.09.002

8. Lustig SL, Kureshi S, Delucchi KL, Iacopino V, Morse SC. Asylum grant rates following medical evaluations of maltreatment among political asylum applicants in the United States. J Immigr Minor Health. 2008;10(1):7-15. doi:10.1007/s10903-007-9056-8

9. Goldfeld AE, Mollica RF, Pesavento BH, Faraone SV. The physical and psychological sequelae of torture. JAMA. 1988;259(18):2725-2729. doi:10.1001/jama.1988.03720180051032

10. Asgary R, Charpentier B, Burnett DC. Socio-medical challenges of asylum seekers prior and after coming to the US.J Immigr Minor Health. 2013;15(5):961-968. doi:10.1007/ s10903-012-9687-2

11. Bischoff A, Bovier PA, Rrustemi I, Gariazzo F, Eytan A, Loutan L. Language barriers between nurses and asylum seekers: their impact on symptom reporting and referral. Soc Sci Med. 2003;57(3):503-512. doi:0.1016/s0277-9536(02)00376-3

\section{(c) 2021 Author(s)}

This is an open-access article distributed under the terms of the Creative Commons Attribution 4.0 International license, which permits anyone to download, copy, distribute, display, or adapt the text without asking for permission, provided that the creator(s) are given full credit.

ISSN 2470-9727 